\title{
Establishment and application of a comprehensive assessment system for cold resistance in Denphal-group Dendrobium cultivars
}

\author{
Shunjiao $\mathrm{Lu}^{1,2}$, Jiaqi $\mathrm{He}^{1}$, Shuangshuang $\mathrm{Yi}^{1,2}$, Yi Liao ${ }^{1,2}$, Chonghui $\mathrm{Li}^{1,2}$, Suiguang Yang ${ }^{1,2}$ and \\ Junmei Yin $1,2,3$ \\ ${ }^{1}$ Tropical Crops Genetic Resources Institute, Chines Academy of Tropical Agricultural Sciences/ Key Laboratory of Crop \\ Gene Resources and Germplasm Enhancement in Southern China, Danzhou, Hainan, China \\ ${ }^{2}$ Hainan Engineering Center of Tropical Ornamental Plant Germplasm Innovation and Utilization, Danzhou, Hainan, China \\ ${ }^{3}$ Haikou Experimental Station, CATAS, Haikou, China
}

\section{Summary}

The objective of this study was to establish a comprehensive assessment system for cold resistance in Denphal-group Dendrobium cultivars, and evaluation of cold resistance of 35 Denphal-group Dendrobium cultivars. The concentration of soluble sugar, soluble protein, free proline, malondialdehyde, chlorophyll, and LT50 of two Denphal-group Dendrobium cultivars were measured under low temperature, and significance analysis, correlation analysis, principal component analysis, and weight analysis of these indicators were conducted, and then a comprehensive assessment system was constructed using the membership function and weight of indicators methods. Secondly, the cold resistance of 35 cultivars was assessed using the comprehensive assessment system. Under our experimental conditions, free proline, malondialdehyde, and chlorophyll were significantly correlated with LT50 at 0.05-level. The principal component analysis and weight analysis indicated that the most significant factor affecting cold resistance was free proline, followed by MDA and chlorophyll, indicating that the three indicators could be used to determine cold resistance in Denphal-group Dendrobium cultivars. The cold resistance evaluation of 35 cultivars showed that the most cold-resistant cultivar was 'Sophie Bonnie', and the most sensitive cultivar was 'More Red $\times$ Thailand'. These results demonstrated that the three indicators of free proline, MDA and chlorophyll could be used to determine cold resistance in Denphal-group Dendrobium cultivars.

Keywords

cold resistance, comprehensive assessment, Denphalgroup Dendrobium, membership function method, physiological indicators

\section{Introduction}

Dendrobium is among the largest genera in flowering plants, with more than 1,200 identified species throughout the world, most of which grow as epiphytes in tropical and subtropical Asia and eastern Australia (Wang et al., 2009). Interest in Dendrobium species is broad, ranging from traditional medicine to ornamental horticulture. In recent years,

\section{Significance of this study}

What is already known on this subject?

- Denphal-group Dendrobium is original in tropical and subtropical regions; low temperature is a key limitation of the expanding cultivation area and inhibiting the industrial development. Selecting and breeding high cold tolerance cultivars is an important method to solve the problem. Comprehensive assessment systems are widely used to assess plant tolerance to abiotic stresses.

What are the new findings?

- The physiology indicators of free proline, MDA and chlorophyll were the most significant factors affecting cold resistance of Denphal-group Dendrobium cultivars, and could be used to determine cold resistance in Denphal-group Dendrobium cultivars. The most cold-resistant cultivar was 'Sophie Bonnie', and the most sensitive cultivar to low temperature was 'More Red $\times$ Thailand' of 35 detected cultivars.

What is the expected impact on horticulture?

- The comprehensive assessment system is useful for Denphal-group Dendrobium germplasm resources cold resistance evaluation. The high cold resistance cultivars could be a potential parent for cold resistance cultivars breeding and expanding cultivation area of Denphal-group Dendrobium.

Dendrobium orchids have become increasingly popular for ornamental use due to their floriferous nature and availability in a wide range of colors, sizes, and shapes. Compared with other flowering plants, Dendrobium flowers also have a relatively long life, lasting several weeks to months.

Although a large number of morphological variations exist in Dendrobium, there are two groups in the genus suited for ornamental sale: the Nobile-group and the Denphal-group. The Nobile-group produces inflorescences and flowers that are distributed along the pseudobulbs, and the Denphal-group produces one or more terminal inflorescences from the pseudobulbs (Cardoso, 2012).

Commercial cultivation of Denphal-group Dendrobium is relatively recent in China and some subtropical regions, 
and Denphal-group cultivars breeding is started late. Thus, Denphal-group Dendrobium cultivars are mostly imported from Thailand or Taiwan, where temperatures are high yearround and are suitable for Denphal-group cultivar growth (Gong et al., 2007). In China and some subtropical regions, however, it becomes cold in the winter, which can cause low temperature damage to plants, including leaves yellowed rapidly, leaf and flower senescence, growth retardation, etc. (He et al., 2016). Due to these challenges, it is necessary to select and breed high cold-tolerant cultivars for China and the subtropical regions having cold winters. However, we are not aware of any research on cold resistance in Denphal-group Dendrobium in these regions.

The aim of this study was to establish a comprehensive assessment system for cold resistance in Denphal-group Dendrobium cultivars, and then to select the cultivars with high cold tolerance for cultivation and high cold tolerance cultivar breeding.

\section{Materials and methods}

\section{Plant materials}

Preliminary evaluation of cold resistance in Denphal-group Dendrobium cultivars in the field (under natural low temperature) found that the cultivar Dendrobium 'Udomsri Beauty' had excellent cold resistance, and 'Sonia Hiasakul' was sensitive to low temperatures (He et al., 2016). Based on these preliminary results, 'Udomsri Beauty' and 'Sonia Hiasakul' were selected to measure the physiological indicators of cold resistance, as well as to establish a comprehensive cold resistance assessment system. The cold resistance of 35 Denphal-group Dendrobium cultivars (including 'Udomsri Beauty' and 'Sonia Hiasakul') were evaluated by the comprehensive cold resistance assessment system.

\section{Methods}

Mature seedlings of 'Udomsri Beauty' and 'Sonia Hiasakul' were placed in a plant growth chamber at $15^{\circ}, 10^{\circ}$ and $5^{\circ} \mathrm{C}$ with $85 \%$ humidity and a $14 / 10 \mathrm{~h}$ light/dark period. The tips of fully extended leaves of three plants were collected at $0,1,2,4,8,24 \mathrm{~h}$ and 2, 4, 8 16-day periods and soluble protein, soluble sugar, malondialdehyde (MDA), free proline, and chlorophyll were measured. The soluble protein concentration was prepared using the Coomassie Brilliant Blue G-250 staining method (Li, 2000), and the anthrone method was used to determine soluble sugar concentration ( $\mathrm{Li}$, 2000). MDA, free proline, and the chlorophyll concentrations were determined based on the methods of Li (Li, 2000). Each treatment and measurement played three replicates.

Additionally, mature seedlings of 'Udomsri Beauty' and 'Sonia Hiasakul' were placed in a plant growth chamber at $10^{\circ}, 5^{\circ}, 0^{\circ}$ and $-5^{\circ} \mathrm{C}$ with $85 \%$ humidity and a $14 / 10 \mathrm{~h}$ light/ dark period. After $12 \mathrm{~h}$, tips of fully extended leaves of three plants were collected, and the relative electric conductivity (REC) of electrolyte leakage, as well as the semi-lethal temperature (LT50), were determined using the methods outlined by Li (Li, 2000). Each treatment and measurement played three replicates.

\section{Statistics}

The mean, standard deviation, significance analysis, correlation analysis, and principal component analysis were all calculated using Duncan's multiple range test (Duncan, 1955) using SPSS 20.0 (SPSS Inc., Chicago, Illinois). Indicators with a significant correlation were extracted to calculate the contribution rate $\left(\mathrm{C}_{\mathrm{i}}\right)$ and weight $\left(\mathrm{W}_{\mathrm{i}}\right)$ with principal component analysis. These above processes were also achieved by SPSS 20.0.

A slightly modified membership function method was used as a comprehensive evaluation of the 35 Denphal-group Dendrobium cultivars, as outlined by Zhang (Zhang et al., 2012) and Wang (Wang et al., 2014). If the correlation between the index and LT50 was positive, the specific membership function value was calculated using Equation 1:

$$
\mathrm{X}_{\mathrm{ij}}=\left(\mathrm{X}-\mathrm{X}_{\min }\right) /\left(\mathrm{X}_{\max }-\mathrm{X}_{\min }\right) \text {. }
$$

If the correlation between the index and LT50 was negative, the specific membership function values were calculated using Equation 2:

$$
\mathrm{X}_{\mathrm{ij}}=\left(\mathrm{X}_{\max }-\mathrm{X}\right) /\left(\mathrm{X}_{\max }-\mathrm{X}_{\min }\right)
$$

where $\mathrm{X}$ is the value of character $\mathrm{j}$ for cultivar $\mathrm{i}, \mathrm{X}_{\min }$ is the minimum value of character $j$ among all measurements of cultivar $\mathrm{i}, \mathrm{X}_{\max }$ is the maximum value of character $\mathrm{j}$ among all measurements of cultivar $\mathrm{i}$, and $\mathrm{X}_{\mathrm{ij}}$ is the membership value of cold resistance of cultivar i and character $\mathrm{j}$. For each cultivar, the integrated index (I) was calculated using the multiplication and addition Equation 3:

$$
\mathrm{I}=\sum\left(\mathrm{W}_{\mathrm{i}} \times \mathrm{X}_{\mathrm{ij}}\right)
$$

The value of 'I' denoted the level of cold resistance, with higher cultivar values indicating increased resistance.

\section{Results}

\section{Optimum temperature and treatment period selection}

The concentrations of soluble protein, soluble sugar, MDA, free proline, and chlorophyll of the two cultivars were shown in Supplemental Tables 1-5. Soluble protein tended to rise, fall, and then rise again in 'Sonia Hiasakul' and 'Udoms-

\begin{tabular}{|c|c|c|c|c|c|c|}
\hline & LT50 & Soluble protein & Soluble sugar & Chlorophyll & Free proline & MDA \\
\hline LT50 & & 0.395 & 0.633 & $-0.880^{* *}$ & $0.947^{* *}$ & $0.958^{* *}$ \\
\hline Soluble protein & 0.449 & & -0.212 & $-0.719^{* *}$ & 0.338 & 0.500 \\
\hline Soluble sugar & $0.875^{* *}$ & 0.523 & & 0.357 & $0.744^{\star *}$ & 0.479 \\
\hline Chlorophyll & $-0.868^{* *}$ & -0.485 & $-0.927^{* *}$ & & $-0.872^{* *}$ & $-0.953^{* *}$ \\
\hline Free proline & $0.947^{\star *}$ & 0.610 & $0.906 * *$ & $-0.833^{*}$ & & 0.930 ** \\
\hline MDA & $0.925^{\star *}$ & 0.623 & $0.885^{* *}$ & $-0.919 * *$ & $0.954^{* *}$ & \\
\hline
\end{tabular}
ri Beauty', with the processing time in same temperature.

TABLE 1. Correlation coefficient matrix of Dendrobium 'Sonia Hiasakul' under low temperature stress.

${ }^{* *}$ Correlation is significant at the 0.01 level (2-tailed).

${ }^{*}$ Correlation is significant at the 0.05 level (2-tailed). 
TABLE 2. Correlation coefficient matrix of Dendrobium 'Udomsri Beauty' under low temperature stress.

\begin{tabular}{lcccccc}
\hline & LT50 & Soluble protein & Soluble sugar & Chlorophyll & Free proline & MDA \\
\hline LT50 & & 0.066 & -0.371 & $-0.983^{* *}$ & $0.985^{* *}$ & $0.770^{* *}$ \\
Soluble protein & -0.556 & & -0.217 & -0.053 & 0.046 & 0.077 \\
Soluble sugar & 0.650 & -0.299 & & 0.227 & -0.370 & 0.072 \\
Chlorophyll & $-0.967^{* *}$ & 0.542 & -0.642 & & $-0.891^{*}$ & $-0.980^{* *}$ \\
Free proline & $0.885^{* *}$ & -0.554 & 0.597 & $-0.966^{*}$ & & $0.758^{*}$ \\
MDA & $0.800^{* *}$ & -0.470 & 0.394 & $-0.917^{* *}$ & $0.949^{* *}$ & \\
\hline
\end{tabular}

** Correlation is significant at the 0.01 level (2-tailed).

${ }^{*}$ Correlation is significant at the 0.05 level (2-tailed).

TABLE 3. Total variance explained of each variety of Denphal-group Dendrobium cultivars.

\begin{tabular}{lccc}
\hline \multirow{2}{*}{ Component } & \multicolumn{3}{c}{ Characteristic vector } \\
\cline { 2 - 4 } & Eigenvalue & Contributiveness (\%) & Cumulative (\%) \\
\hline 1 & 2.569 & 51.386 & 51.386 \\
2 & 1.837 & 36.741 & 88.127 \\
3 & 0.448 & 9.762 & 97.889 \\
4 & 0.106 & 2.111 & 100.000 \\
\hline
\end{tabular}

TABLE 4. Capacity and weight of cold resistance indicator of Denphal-group Dendrobium cultivars.

\begin{tabular}{|c|c|c|c|c|}
\hline \multirow[b]{2}{*}{ Index } & \multicolumn{2}{|c|}{ Load } & \multirow[b]{2}{*}{$\mathrm{Fi} \times \mathrm{Y} 1+\mathrm{Fi} \times \mathrm{Y} 2$} & \multirow[b]{2}{*}{ Weight } \\
\hline & $\begin{array}{c}\text { The first } \\
\text { principal component }\end{array}$ & $\begin{array}{c}\text { The second } \\
\text { principal component }\end{array}$ & & \\
\hline Chlorophyll & 0.875 & 0.028 & 0.4599 & 0.1599 \\
\hline Free proline & 0.872 & -0.45 & 0.6132 & 0.2132 \\
\hline MDA & -0.559 & -0.805 & 0.6166 & 0.2144 \\
\hline LT50 & 0.570 & 0.672 & 0.5398 & 0.1877 \\
\hline
\end{tabular}

The concentration of soluble sugar, MDA, and free proline increased with processing time in both cultivars, and chlorophyll decreased with processing time. All indicators showed a significant difference to control at $5^{\circ} \mathrm{C}$ for $8 \mathrm{~d}$, thus, the critical treatment temperature and period appears to be $5^{\circ} \mathrm{C}$ and $8 \mathrm{~d}$.

\section{Correlation analysis of cold resistance indicators}

The correlation analysis of six cold hardiness indicators (Table 1) found that for 'Sonia Hiasakul', soluble sugar, MDA, and free proline were positively correlated with LT50 $(p \leq 0.01)$, and that chlorophyll was negatively correlated with LT50, soluble protein, MDA $(p \leq 0.01)$ and free proline $(p \leq 0.05)$. For 'Udomsri Beauty', MDA and free proline were positively correlated with LT50 $(p \leq 0.01)$ and chlorophyll was negatively correlated with LT50 $(p \leq 0.01)$. These results showed that the correlations between LT50 and MDA, free proline, and chlorophyll were conspicuous in both 'Sonia Hiasakul' and 'Udomsri Beauty'. Thus, using MDA, free proline and chlorophyll as indicators to assess cold resistance should provide a more accurate and robust assessment of cold resistance of Denphal-group Dendrobium cultivars.

\section{Principal component analysis of cold resistance indices}

The results from principal component analysis showed that the contribution rates of the two principal components were about $65.25 \%$ and $20.76 \%$ (Table 2), and that the cumulative contribution rate reached $86.01 \%$, accounting for $86.01 \%$ of the cold resistance of included Denphal-group Dendrobium cultivars. The values of the eigenvector of princi- pal components were $3.26,1.04,0.50$ and 0.20 , respectively. Results show that only the first and second eigenvector values in the principal components were greater than 1 . Thus, the first and second principal components were extracted for subsequent studies. The weight $\left(\mathrm{W}_{\mathrm{i}}\right)$ of LT50, MDA, free proline, and chlorophyll were calculated by the capacity (F) and contribution value (Y) of each index in the first and second principal components. Result showed that the most significant factor affecting cold resistance was free proline, followed by MDA, and lastly, by chlorophyll.

\section{Comprehensive assessment of cold resistance of Denphal-group Dendrobium cultivars}

A comprehensive assessment of the cold resistance of cultivars was conducted using the index membership value and index weight of each cultivar. The results showed that the 35 Denphal-group Dendrobium cultivars could be divided into seven classes according to membership value (Table 4). The highest value of 0.818 represented one cultivar, 'Sophie Bonnie'. The second class contained five cultivars and values ranged from 0.700 and 0.799 . The third class contained six cultivars and values ranged from 0.600 and 0.699 . The fourth class contained 11 cultivars and values were between 0.500 and 0.599. The fifth and sixth classes contained seven and three cultivars, respectively, and values ranged from 0.400 to 0.499 and from 0.300 to 0.399 , respectively.

The comprehensive assessment showed that the 35 Denphal-group Dendrobium cultivars could be divided into six classes (Table 5). The strongest cold resistance class had an index value above 0.600 and contained two cultivars, 'Sophie 
Bonnie' and 'Salaya Pink'. The second strongest cold resistant class had index values from 0.500 to 0.599 and contained seven cultivars. The medium cold resistance class had index values from 0.400 to 0.499 and contained thirteen cultivars. Index values from 0.300 to 0.399 contained eight cultivars and were considered to have low cold resistance. The class with weak cold resistance had index values from 0.200 to 0.299 and was composed of four cultivars. Lastly, the least cold resistance class produced index values less than 0.200 and represented only one cultivar, 'More Red × Thailand'.

\section{Discussion}

Plant adaption to cold stress is a gradual process of morphological, physiological, and biochemical variation accompanied by the accumulation of particular substances such as soluble sugars, soluble protein, free proline, unsaturated fatty acids, and membrane lipid peroxide (Gusta et al., 2003; Strand et al., 1997; Wang, 1987; Zhang et al., 2012). However, the factors affecting plant cold resistance are mul- tifaceted, and the physiological process of plant adaptation to cold stress is extremely complex. Thus, evaluating plant cold resistance by a single index or multiple separate indicators may cause the one-sidedness and deviation. A comprehensive assessment which considers multiple indicators is better able to account for the cold resistance of plants. Such assessments have been used in other crops, such as grape (Zhang et al., 2012), herbaceous peony (Wang et al., 2014), hybrid hazelnut (Lv et al., 2008), Cotton (Wu et al., 2012), Zenia insignis (Liu et al., 2007), chrysanthemum (Xu et al., 2009), apricot (Ai et al., 2013), etc.

Under cold stress, soluble sugars can function as protective substances which increase the osmotic potential of the cytoplasm and lower the freezing temperature, and are positively correlated with cold hardiness (Gusta et al., 2003; Wang, 1987; Zhang et al., 2012). Our study showed that soluble sugars were accumulated in leaves of Denphal-group Dendrobium cultivars under low temperature, and were significantly correlated with LT50 in cold sensitive cultivar

TABLE 5. Average membership degree of cold resistance indicators of Denphal-group Dendrobium cultivars.

\begin{tabular}{|c|c|c|c|c|}
\hline Cultivars & Chlorophyll & MDA & Free proline & $\begin{array}{c}\text { Average membership } \\
\text { degree }\end{array}$ \\
\hline Dendrobium Sophie Bonnie & 0.6226 & 0.8883 & 0.9421 & 0.8177 \\
\hline Dendrobium Salaya Pink & 0.6358 & 0.8834 & 0.7860 & 0.7684 \\
\hline Dendrobium Enobi Purple & 0.5912 & 1.0000 & 0.6520 & 0.7477 \\
\hline Dendrobium Siriratana $\times$ D. Chao Praya Gem & 1.0000 & 0.2343 & 0.9623 & 0.7322 \\
\hline Dendrobium Burana Sundae × D. Black Cat & 0.5673 & 0.7215 & 0.8323 & 0.7070 \\
\hline Dendrobium Bigibbum & 0.4309 & 0.9987 & 0.6818 & 0.7038 \\
\hline Dendrobium Salaya $\times$ D. Sriprathum Red & 0.4377 & 0.8975 & 0.6417 & 0.6590 \\
\hline Dendrobium Madame Lucy & 0.5872 & 0.8003 & 0.5446 & 0.6440 \\
\hline Dendrobium New Stripes Delite & 0.4837 & 0.8613 & 0.5587 & 0.6346 \\
\hline Dendrobium X1 & 0.2660 & 0.8126 & 0.7893 & 0.6226 \\
\hline Dendrobium Marilyn Monroe & 0.3435 & 0.6620 & 0.8209 & 0.6088 \\
\hline Dendrobium Burana Green & 0.6564 & 0.2546 & 0.8985 & 0.6032 \\
\hline Dendrobium Burana Charming $\times D$. Earsakul & 0.5729 & 0.6937 & 0.5273 & 0.5980 \\
\hline Dendrobium Lady Lisa & 0.5859 & 0.8387 & 0.3656 & 0.5967 \\
\hline Dendrobium Udomsri Beauty & 0.4954 & 0.6395 & 0.6305 & 0.5884 \\
\hline Dendrobium Burana Stripe & 0.3527 & 0.7698 & 0.6398 & 0.5874 \\
\hline Dendrobium Nopporn White Diamond & 0.4936 & 0.8201 & 0.3789 & 0.5642 \\
\hline Dendrobium Rainbow Compactum & 0.3189 & 0.7755 & 0.5827 & 0.5591 \\
\hline Dendrobium Sirin Peach & 0.3281 & 0.5369 & 0.7750 & 0.5467 \\
\hline Dendrobium X2 & 0.5224 & 0.7195 & 0.3884 & 0.5434 \\
\hline Dendrobium Sirin Peach & 0.3300 & 0.6471 & 0.6104 & 0.5292 \\
\hline Dendrobium New Aridang Blue Mutation & 0.5220 & 0.4828 & 0.5616 & 0.5221 \\
\hline Dendrobium NPR863 & 0.5331 & 0.6201 & 0.3525 & 0.5019 \\
\hline Dendrobium Angel Blue & 0.4319 & 0.5824 & 0.4584 & 0.4909 \\
\hline Dendrobium NPR727 & 0.3324 & 0.5145 & 0.6046 & 0.4839 \\
\hline Dendrobium Popeye & 0.4884 & 0.6901 & 0.2481 & 0.4755 \\
\hline Dendrobium Aridang & 0.4537 & 0.5661 & 0.3662 & 0.4620 \\
\hline Dendrobium Mary Green & 0.2832 & 0.6682 & 0.4241 & 0.4585 \\
\hline Dendrobium Liberty White & 0.4105 & 0.6254 & 0.2945 & 0.4435 \\
\hline Dendrobium Sonia Hiasakul & 0.5148 & 0.6559 & 0.1327 & 0.4345 \\
\hline Dendrobium NPR844 & 0.6562 & 0.4404 & 0.0913 & 0.3959 \\
\hline Dendrobium NPR699 & 0.5994 & 0.0080 & 0.5777 & 0.3950 \\
\hline Dendrobium Sunny Red & 0.2730 & 0.3679 & 0.3753 & 0.3387 \\
\hline Dendrobium Burana Jade & 0.0000 & 0.0114 & 0.7621 & 0.2578 \\
\hline Dendrobium More Red $\times D$. Thailand & 0.2966 & 0.0000 & 0.4737 & 0.2568 \\
\hline
\end{tabular}


'Sonia Hiasakul', a finding which has also been reported in grape (Cao et al., 2010; Zhang et al., 2012), Zenia insignis (Liu et al., 2007), Cymbidium hybridium (Zhou et al., 2017), chewing cane cultivars (Chen et al., 2018), and peach (Feng et al., 2018). However, the soluble sugars showed no significant correlation with LT50 in the cold tolerant cultivar 'Udomsri Beauty', and was similar to herbaceous peony (Wang et al., 2014), azalea cultivars (Liu et al., 2016) and Ilex centrochinensis (Hu et al., 2018). This observation indicates that increases in the soluble sugar content is a low temperature stress response and can be used to evaluate cold resistance in some plants, but not all.

Soluble protein content also increases under cold stress, due to increased rates of synthesis or the degradation of high molecular weight material (Wang, 1987). As soluble protein can increase the proportion of bound intracellular water, there is an associated reduction in the availability of free water. The concentrations of soluble proteins are strongly associated and both tend to increase the water-retention capacity of the protoplast (Dionne et al., 2001). Our study showed that under cold stress, soluble proteins accumulated in the leaves of cultivars, but showed no significant correlation with LT50, similar to research with azalea (Liu et al., 2016), pineapple (Lu et al., 2010), potato (Yang and Guo, 2016), Prunus domestica (Abula et al., 2017).

MDA is the membranous peroxidation product in the organ; when the plant organs are undergoing adverse conditions, its accumulation can reflect the response strength of the plants to adverse conditions (Tabassum et al., 2014). The accumulation of MDA is considered to reflect the physiological state of plant membrane lipids under cold stress (Imahori et al., 2008). Our study showed that MDA significantly correlated with LT50 in the Denphal-group Dendrobium cultivars, that is consistent with studies with most plants, including peach (Feng et al., 2018), Ilex centrochinensis (Hu et al., 2018), chewing cane cultivars (Chen et al., 2018), Cymbidium hybridium (Zhou et al., 2017), herbaceous peony (Wang et al., 2014), grape (Cao et al., 2010; Zhang et al., 2012). Moreover,

TABLE 6. Integrated index of cold resistance indicator of Denphal-group Dendrobium cultivars.

\begin{tabular}{|c|c|c|c|c|}
\hline Cultivars & Chlorophyll & MDA & Free proline & Integrated index \\
\hline Dendrobium Sophie Bonnie & 0.1412 & 0.2491 & 0.2525 & 0.6428 \\
\hline Dendrobium Salaya Pink & 0.1442 & 0.2478 & 0.2107 & 0.6026 \\
\hline Dendrobium Enobi Purple & 0.1341 & 0.2805 & 0.1747 & 0.5893 \\
\hline Dendrobium Bigibbum & 0.0977 & 0.2801 & 0.1827 & 0.5606 \\
\hline Dendrobium Burana Sundae $\times$ D. Black Cat & 0.1286 & 0.2024 & 0.2231 & 0.5541 \\
\hline Dendrobium Siriratana $\times D$. Chao Praya Gem & 0.2268 & 0.0657 & 0.2579 & 0.5504 \\
\hline Dendrobium Salaya × D. Sriprathum Red & 0.0993 & 0.2517 & 0.1720 & 0.5230 \\
\hline Dendrobium Madame Lucy & 0.1332 & 0.2245 & 0.1459 & 0.5036 \\
\hline Dendrobium New Stripes Delite & 0.1097 & 0.2416 & 0.1497 & 0.5010 \\
\hline Dendrobium X1 & 0.0603 & 0.2279 & 0.2115 & 0.4998 \\
\hline Dendrobium Marilyn Monroe & 0.0779 & 0.1857 & 0.2200 & 0.4836 \\
\hline Dendrobium Burana Stripe & 0.0800 & 0.2159 & 0.1715 & 0.4674 \\
\hline Dendrobium Lady Lisa & 0.1328 & 0.2352 & 0.0980 & 0.4660 \\
\hline Dendrobium Burana Charming $\times D$. Earsakul & 0.1299 & 0.1946 & 0.1413 & 0.4658 \\
\hline Dendrobium Burana Green & 0.1488 & 0.0714 & 0.2408 & 0.4611 \\
\hline Dendrobium Udomsri Beauty & 0.1123 & 0.1794 & 0.1690 & 0.4607 \\
\hline Dendrobium Rainbow Compactum & 0.0723 & 0.2175 & 0.1562 & 0.4460 \\
\hline Dendrobium Nopporn White Diamond & 0.1119 & 0.2300 & 0.1016 & 0.4435 \\
\hline Dendrobium Sirin Peach & 0.0744 & 0.1506 & 0.2077 & 0.4327 \\
\hline Dendrobium $\mathrm{X} 2$ & 0.1185 & 0.2018 & 0.1041 & 0.4243 \\
\hline Dendrobium Nopporn Pink & 0.0748 & 0.1815 & 0.1636 & 0.4199 \\
\hline Dendrobium New Aridang Blue Mutation & 0.1184 & 0.1354 & 0.1505 & 0.4043 \\
\hline Dendrobium NPR863 & 0.1209 & 0.1739 & 0.0945 & 0.3893 \\
\hline Dendrobium Angel Blue & 0.0979 & 0.1633 & 0.1229 & 0.3842 \\
\hline Dendrobium NPR727 & 0.0754 & 0.1443 & 0.1620 & 0.3817 \\
\hline Dendrobium Popeye & 0.1107 & 0.1935 & 0.0665 & 0.3708 \\
\hline Dendrobium Mary Green & 0.0642 & 0.1874 & 0.1137 & 0.3653 \\
\hline Dendrobium Aridang & 0.1029 & 0.1588 & 0.0981 & 0.3598 \\
\hline Dendrobium Liberty White & 0.0931 & 0.1754 & 0.0789 & 0.3474 \\
\hline Dendrobium Sonia Hiasakul & 0.1167 & 0.1840 & 0.0356 & 0.3363 \\
\hline Dendrobium NPR844 & 0.1488 & 0.1235 & 0.0245 & 0.2968 \\
\hline Dendrobium NPR699 & 0.1359 & 0.0022 & 0.1548 & 0.2930 \\
\hline Dendrobium Sunny Red & 0.0619 & 0.1032 & 0.1006 & 0.2657 \\
\hline Dendrobium Burana Jade & 0.0000 & 0.0032 & 0.2043 & 0.2075 \\
\hline Dendrobium More Red $\times D$. Thailand & 0.0673 & 0.0000 & 0.1270 & 0.1942 \\
\hline
\end{tabular}


weight analysis showed that MDA was the second most significant factor affecting cold resistance, suggesting that it can be used as an effective indicator for the cold resistance evaluation of Denphal-group Dendrobium cultivars.

Free proline can help maintain osmotic equilibrium between the symplast and apoplast in plant cells, and thus, proline aids in preventing low-temperature damage by maintaining the functional integrity of the membrane (Dionne et al., 2001). Therefore, under cold stress, cold-resistant plants would accumulate free proline more than the less resistant ones. In our study, the content of free proline increased under low temperature and was significantly correlated with LT50. We showed that free proline was the most significant factor affecting cold resistance in cultivars, a conclusion that has also been reached in experiments with peach (Feng et al., 2018), grape (Cao et al., 2010; Zhang et al., 2012), herbaceous peony (Wang et al., 2014). Our research indicated that free proline responds to low temperatures, and was an effective indicator for cold resistance evaluation of Denphal-group Dendrobium cultivars.

Chloroplasts are extremely sensitive to low temperature reactions and under low temperature, the chloroplasts ultrastructure is damaged, causing photosynthetic pigment degradation and a decline in the chlorophyll content. The variation of chlorophyll content is an important indicator of crop tolerance to cold. Our research showed that the chlorophyll content of Denphal-group Dendrobium cultivars declines under low temperatures. Moreover, chlorophyll content was significantly correlated with the LT50, a relationship that had also been shown in flue-cured tobacco varieties (Chen et al., 2008) and Ilex centrochinensis (Hu et al., 2018). In addition, the principal component analysis and the weight analysis we conducted shows that chlorophyll was the third most significant factor affecting cold resistance in Denphal-group Dendrobium cultivars. Therefore, chlorophyll also could be used as an effective indicator for cold resistance evaluation of Denphal-group Dendrobium cultivars.

In conclusion, our results showed that free proline, MDA, and chlorophyll are important indicators of cold resistance in Denphal-group Dendrobium cultivars. Furthermore, we showed that soluble sugar and soluble proteins respond to cold stress, but due to variation among cultivars, were not suitable to be used as a cold resistance evaluation indicator for Denphal-group Dendrobiums.

We provided evidence of variation in cold tolerance among Denphal-group Dendrobium cultivars. Our data offered a valuable resource for cold resistance breeding in Denphal-group Dendrobium, and our results indicated that the cultivar 'Sophie Bonnie' had the most cold resistance, followed by 'Salaya Pink', 'Enobi Purple', and 'Bigibbum'. The hybrids 'Burana Sundae $\times$ Black Cat', 'Siriratana' $\times$ 'Chao Praya Gem', and 'Salaya $\times$ Sriprathum Red' also had high cold resistance. These cultivars were the important resources for Denphal-group Dendrobium cold resistance cultivar breeding.

\section{Conclusion}

Under our experimental conditions, free proline, malondialdehyde, and chlorophyll were significantly correlated with LT50 at 0.05 level, and were the most significant physiology indicators affecting cold resistance between soluble sugar, soluble protein, free proline, malondialdehyde, chlorophyll. Within 35 cultivars, the most cold-resistant cultivar was 'Sophie Bonnie', and the most sensitive cultivar was 'More Red $\times$ Thailand'.

\section{Acknowledgments}

This research was financially supported by the National Key Research and Development Program of China, Mechanism analysis on major flowers coloration (Grant no. 2018YFD1000405), the Hain-an Natural Science Fund Project (Grant no. 320RC722), the Central Public-interest Scientific Institution Basal Research Fund for Chinese Academy of Tropical Agricultural Sciences (Grant no. 1630032017021, 1630032017024 and 1630032020035$).$

\section{References}

Abula, N., Tiemuer, A., Tuoheti, B., and Abulitifu, Y. (2017). Comprehensive evaluation of cold resistance of three rootstocks of P. domestica L. South China Fruits 47(2), 13-20.

Ai, P.F., Jin, X.J., Jin, Z.Z., Yan, F.Q., and Li, K.W. (2013). Evaluation of cold-resistance physiological indices of kernel apricot. J. Hebei Univ. Sci. Technol. 34(1), 48-53, 59.

Cao, J.D., Chen, B.H., Wang, L.J., Mao, J., and Zhao, X. (2010). Cold resistance indexes identification and comprehensive evaluation of grape varieties. Acta Bot. Bor-Occid. Sin. 30(11), 2232-2239.

Cardoso, J.C. (2012). Dendrobium 'Brazilian Fire 101' - New option of color of flowers for the orchid market. Hortic. Bras. 30, 561-564. https://doi.org/10.1590/S0102-05362012000300035.

Chen, M.H., Cheng, S.P., Zhang, Z.L., and Zhang, B.Q. (2018). Physiological response and cold resistance evaluation of seedlings of different chewing cane cultivars to low temperature. J. South China Agric. Univ. (Nat. Sci.) 39(2), 40-46.

Chen, W.G., Li, Y.L., Zhou, J.H., and Yang, H.Q. (2008). Study on chilling tolerance and related physiological indices of flue-cured tobacco varieties. Chin. Tob. Sci. 29(3), 39-42, 47.

Dionne, J., Castonguay, Y., Nadeau, P., and Desjardins, Y. (2001). Freezing tolerance and carbohydrate changes during cold acclimation of green-type annual Bluegrass (L.) ecotypes. Cropence 41, 443-451. https://doi.org/10.2135/cropsci2001.412443x.

Feng, Y.H., Yue, C., and Fan, B.G. (2018). Evaluation of cold resistance of some peach varieties in Shanxi Province. Jiangsu Agric. Sci. 46, 139-142.

Gong, J.Y., Yu, X.B., and Xu, D.P. (2007). Current status of cultivation and management of Dendrobium in Hainan. Tropical Forestry 35(2), 39-42.

Gusta, L.V., Wisniewski, M., Nesbitt, N.T., and Tanino, K.T. (2003). Factors to consider in artificial freeze tests. Acta Hortic. Sin. 618, 493-507. https://doi.org/10.17660/ActaHortic.2003.618.58.

He, J.Q., Lu, S.J., Huang, S.H., Zhang, D.X., and Yin, J.M. (2016). Evaluation on cold resistance of Dendrobium in the field under natural low temperature. J. Anhui Agric. Sci. 44(30), 12-15.

Hu, M.J., Jin, X.L., Zeng, W., Cai, M.Y., and Yu, Z.Y. (2018). Identification and comprehensive evaluation of cold resistance indexes of Ilex centrochinensis. J. Central South Univ. Forestry Technol. 38(5), 59-64.

Imahori, Y., Takemura, M., and Bai, J.H. (2008). Chilling-induced oxidative stress and antioxidant responses in mume (Prunus mume) fruit during low temperature storage. Postharvest Biol. Technol. 49(1), 54-60. https://doi.org/10.1016/j.postharvbio.2007.10.017.

Li, H.S. (2000). The Experimental Principles and Techniques of Plant Physiology and Biochemistry (Beijing: Higher Education Press).

Liu, B., Cao, S., Zhou, H., and Xia, Y.P. (2016). A study on cold tolerance difference and its mechanisms in Azalea cultivars. Acta Hortic. Sin. 43(2), 295-306.

Liu, X.H., He, X.Y., Su, D.M., Wei, L., Yuan, D.Y., and Wang, J.F. (2007). Establishment and application of integrated assessment system on cold resistance of different Zenia insignis provenances. Sci. Silv. Sin. 43(10), 45-50. 
Lu, X.H., Ye, C.H., Sun, D.Q., Li, Y.H., Shi, W.Q., and Sun, G.M. (2010). Evaluation of cold tolerance on 10 Pineapple germplasms under low temperature stress. Chin. J. Trop. Crops 33(11), 1937-1940.

Lv, Y.D., Dong, F.X., Wang, G.X., and Wang, Q.C. (2008). Establishment and application of integrated assessment system on cold resistance of hybrid Hazelnut. Sci. Silv. Sin. 44(9), 31-35.

Strand, A., Hurry, V., Gustafsson, P., and Gardestrom, P. (1997). Development of Arabidopsis thaliana leaves at low temperatures releases the suppression of photosynthesis and photosynthetic gene expression despite the accumulation of soluble carbohydrates. Plant J. 12, 605-614. https://doi.org/10.1046/j.1365-313X.1997.00583.x.

Tabassum, N., Rafique, U., Balkhair, K.S., and Ashraf, M.A. (2014). Chemodynamics of methyl parathion and ethyl parathion: Adsorption models for sustainable agriculture. Biomed. Res. Int. 2014(831989), 1-8. https://doi.org/10.1155/2014/831989.

Wang, H.Z., Feng, S.G., Lu, J.J., Shi, N.N., and Liu, J.J. (2009). Phylogenetic study and molecular identification of 31 Dendrobium species using inter-simple sequence repeat (ISSR) markers. Sci. Hortic. (Amsterdam) 122, 440-447. https://doi.org/10.1016/j. scienta.2009.06.005.

Wang, Q., Cheng, T.R., Yu, X.N., Silva, J.A.T.D., and Byrne, D.H. (2014). Physiological and biochemical responses of six herbaceous peony cultivars to cold stress. S. Afr. J. Bot. 94, 140-148. https://doi. org/10.1016/j.sajb.2014.05.012.

Wang, R.F. (1987). The kinds of plant hardiness criteria and their application. Plant Physiol. Comm. 3, 49-55.

Wu, H., Hou, L.L., Zhou, Y.F., Fan, Z.C., Shi, J.Y., Aliyan, R., and Zhang, J.S. (2012). Analysis of chilling-tolerance and determination of chillingtolerance evaluation indicators in cotton of different genotypes. Acta Hortic. Sin. 45(9), 1703-1713.
Xu, Y., Chen, Y., Chen, F.D., and Chen, S.M. (2009). Analysis of coldtolerance and determination of cold-tolerance evaluation indicators in Chrysanthemum. Acta Hortic. Sin. 42(3), 974-981.

Yang, H.J., and Guo, H.C. (2016). Physiological responds of potato seedlings to low temperature stress and comprehensive evaluation on their cold tolerance. Southwest China J. Agric. Sci. 29(11), 25602567.

Zhang, J., Wu, X., Niu, R., Liu, Y., Liu, N., Xu, W., and Wang, Y. (2012) Cold-resistance evaluation in 25 wild grape species. Vitis $51,153-$ 160.

Zhou, G.Y., Wang, S.Q., Chen, Q.R., Xu, J.X., and Wang, C.C. (2017). Cold resistance indexes identification and comprehensive evaluation of 8 Cymbidium hybridium. J. Fujian. Agric. For. Univ. 46(1), 37-42.

Received: Sep. 12, 2019

Accepted: Nov. 21, 2019

Addresses of authors:

Shunjiao $\mathrm{Lu}^{1,2}$, Jiaqi $\mathrm{He}^{1}$, Shuangshuang $\mathrm{Yi}^{1,2}$, Yi Liao ${ }^{1,2}$, Chonghui Li ${ }^{1,2}$, Suiguang Yang ${ }^{1,2}$ and Junmei Yin ${ }^{1,2, *}$

${ }^{1}$ Tropical Crops Genetic Resources Institute, Chinese Academy of Tropical Agricultural Sciences/Key Laboratory of Crop Gene Resources and Germplasm Enhancement in Southern China, Danzhou, Hainan 571737, China

${ }^{2}$ Hainan Engineering Center of Tropical Ornamental Plant Germplasm Innovation and Utilization, Danzhou, Hainan 571737, China

${ }^{3}$ Haikou Experimental Station, CATAS, Haikou 57010, China * Corresponding author; E-mail: yinjunmei2011@sina.com

\section{SUPPLEMENTAL INFORMATION}



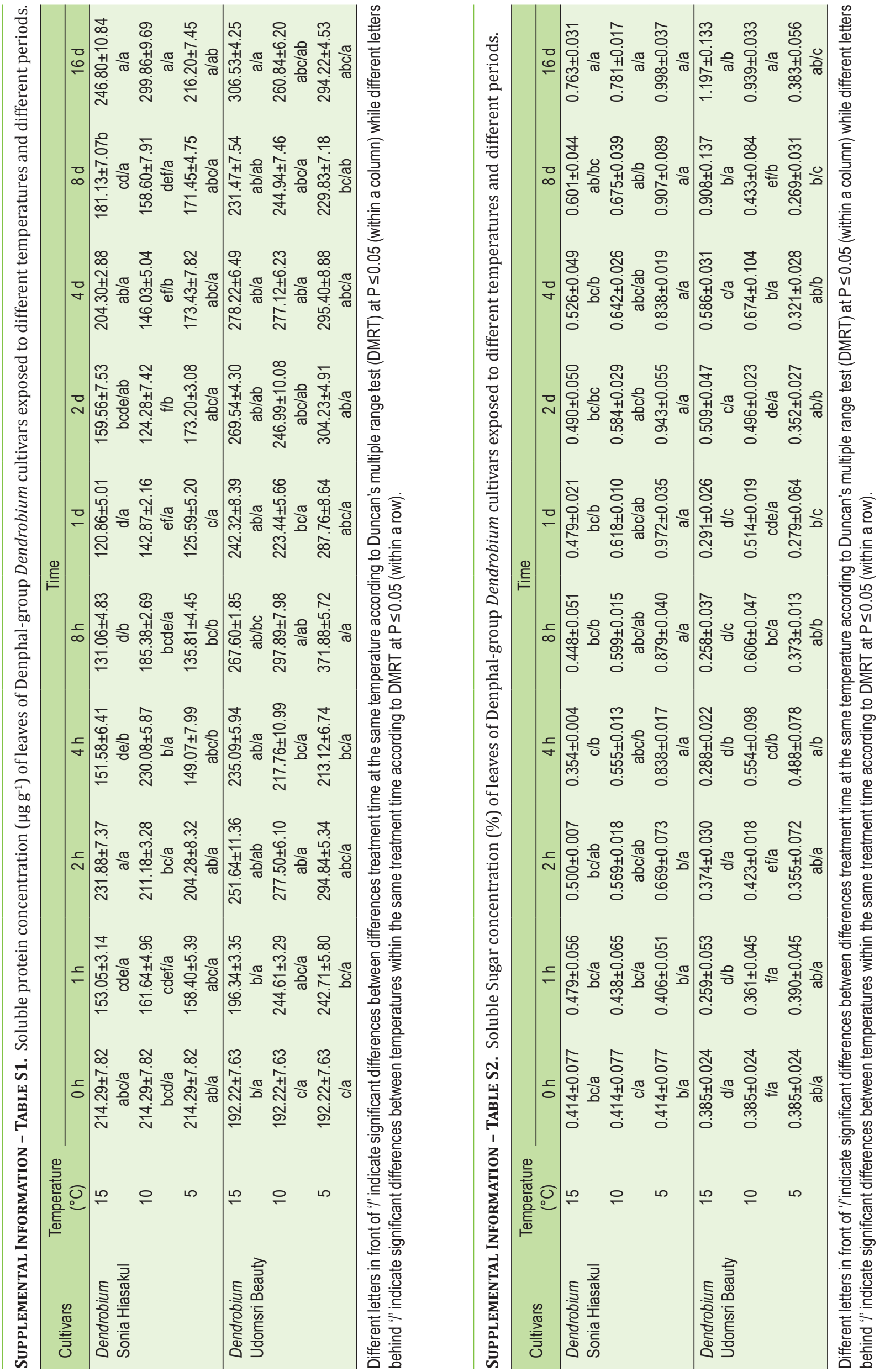

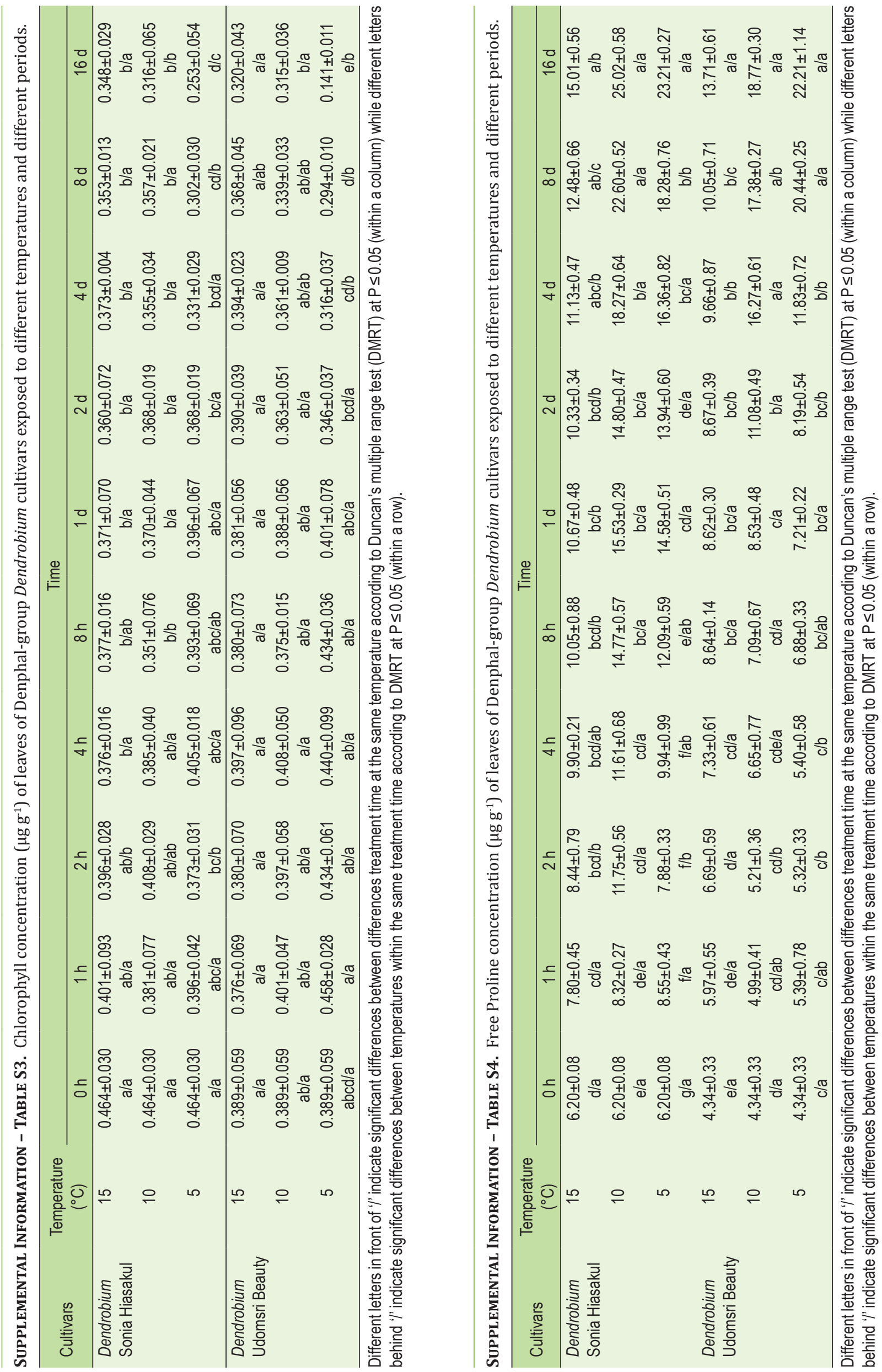


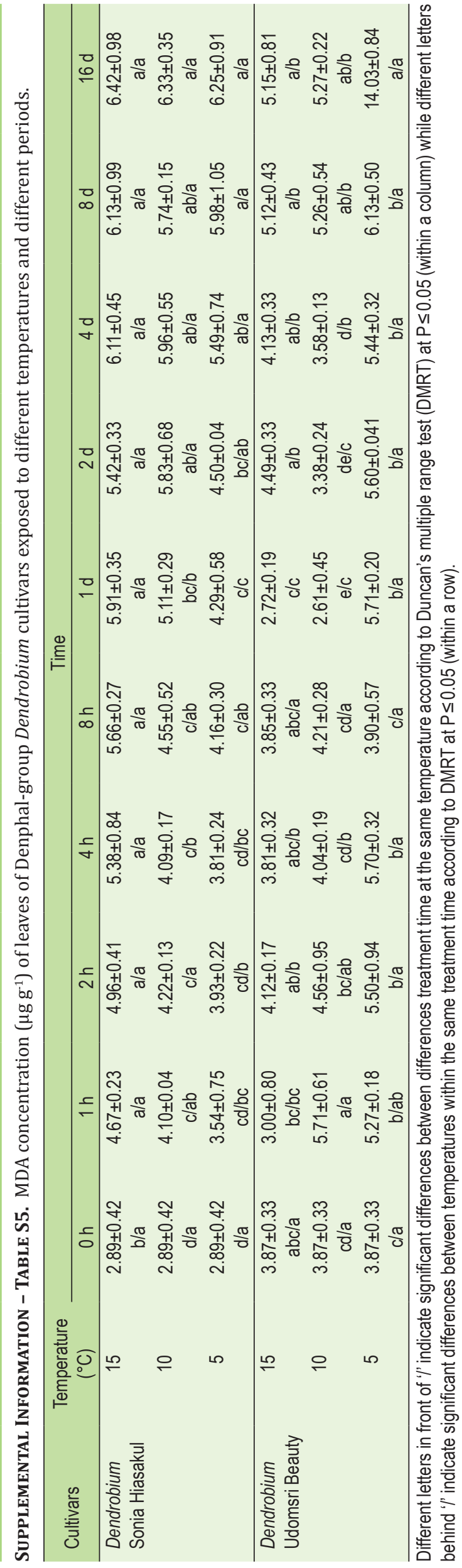


SUPPLEMENTAL INFORMATION - TABLE S6. Logistic equation fitting variance parameters and LT50 of Dendrobium accessions.

\begin{tabular}{|c|c|c|c|c|}
\hline \multirow{2}{*}{ Cultivars } & \multicolumn{2}{|c|}{ Parameter equation } & \multirow{2}{*}{ LT50 } & \multirow{2}{*}{$\mathrm{R} 2$} \\
\hline & a & $b$ & & \\
\hline Dendrobium Sophie Bonnie & 1.5487 & -0.2139 & -2.0452 & $0.961^{*}$ \\
\hline Dendrobium Salaya Pink & 1.2655 & -0.2064 & -1.1408 & $0.951^{*}$ \\
\hline Dendrobium Enobi Purple & 1.1757 & -0.1535 & -1.0545 & $0.972^{*}$ \\
\hline Dendrobium Bigibbum & 1.2382 & -0.2135 & -1.0006 & $0.967^{*}$ \\
\hline Dendrobium Salaya $\times D$. Sriprathum Red & 1.1903 & -0.1773 & -0.9823 & $0.899^{*}$ \\
\hline Dendrobium Burana Sundae $\times D$. Black Cat & 1.1314 & -0.1579 & -0.7819 & $0.980^{\star *}$ \\
\hline Dendrobium X1 & 0.9861 & -0.2126 & 0.0656 & $0.981^{* *}$ \\
\hline Dendrobium Madame Lucy & 0.9698 & -0.2396 & 0.1279 & $0.983^{* *}$ \\
\hline Dendrobium New Stripes Delite & 0.8909 & -0.2473 & 0.4674 & $0.995^{\star *}$ \\
\hline Dendrobium Burana Charming $\times D$. Earsakul & 0.9123 & -0.1875 & 0.4895 & $0.976^{*}$ \\
\hline Dendrobium Burana Stripe & 0.8033 & -0.2077 & 1.0547 & $0.945^{*}$ \\
\hline Dendrobium Burana Green & 0.7939 & -0.2048 & 1.1269 & $0.913^{*}$ \\
\hline Dendrobium NPR727 & 0.7405 & -0.2230 & 1.3472 & $0.987^{* *}$ \\
\hline Dendrobium New Aridang Blue Mutation & 0.6587 & -0.1941 & 2.1508 & $0.949^{*}$ \\
\hline Dendrobium Angel Blue & 0.5643 & -0.2567 & 2.2287 & $0.937^{*}$ \\
\hline Dendrobium NPR863 & 0.5869 & -0.2309 & 2.3075 & $0.99^{* *}$ \\
\hline Dendrobium X2 & 0.5428 & -0.2518 & 2.4267 & $0.960^{*}$ \\
\hline Dendrobium Pop Eye & 0.5511 & -0.2422 & 2.4610 & $0.999 * *$ \\
\hline Dendrobium Aridang & 0.4495 & -0.2990 & 2.6747 & $0.938^{*}$ \\
\hline Dendrobium Mary Green & 0.3202 & -0.3810 & 2.9887 & $0.989^{* *}$ \\
\hline Dendrobium Nopporn Pink & 0.4501 & -0.2659 & 3.0019 & $0.974^{*}$ \\
\hline Dendrobium Siriratana $\times$ D. Chao Praya Gem & 0.3723 & -0.3262 & 3.0290 & $0.973^{*}$ \\
\hline Dendrobium Liberty White & 0.3881 & -0.2967 & 3.1897 & $0.975^{*}$ \\
\hline Dendrobium NPR844 & 0.3433 & -0.3342 & 3.1989 & $0.957^{*}$ \\
\hline Dendrobium Rainbow Compactum & 0.2975 & -0.3647 & 3.3242 & $0.992^{* *}$ \\
\hline Dendrobium Udomsri Beauty & 0.2530 & -0.3990 & 3.4470 & $0.902^{* *}$ \\
\hline Dendrobium Lady Lisa & 0.1832 & -0.4525 & 3.7513 & $0.993^{* *}$ \\
\hline Dendrobium More Red $\times D$. Thailand & 0.3032 & -0.2700 & 4.4206 & $0.961^{*}$ \\
\hline Dendrobium Marilyn Monroe & 0.2759 & -0.2894 & 4.4498 & $0.936^{*}$ \\
\hline Dendrobium Sirin Peach & 0.2154 & -0.3333 & 4.6060 & $0.970^{*}$ \\
\hline Dendrobium Sunny Red & 0.2319 & -0.3139 & 4.6562 & $0.837^{*}$ \\
\hline Dendrobium Burana Jade & 0.1118 & -0.4371 & 5.0138 & $0.919^{*}$ \\
\hline Dendrobium Nopporn White Diamond & 0.1431 & -0.3716 & 5.2307 & $0.978^{*}$ \\
\hline Dendrobium Sonia Hiasakul & 0.2140 & -0.2540 & 6.0710 & $0.9438^{*}$ \\
\hline Dendrobium NPR699 & 0.1670 & -0.2806 & 6.3785 & $0.957^{\star}$ \\
\hline
\end{tabular}

